\title{
THREE-DECADE PROGRAMMABLE FULLY DIFFERENTIAL LINEAR OTA
}

\author{
Yunbin Deng, Shantanu Chakrabartty and Gert Cauwenberghs \\ Department of Electrical and Computer Engineering \\ Johns Hopkins University, Baltimore MD 21218 \\ \{yunbin, shantanu, gert $\} @ j h u . e d u$
}

\begin{abstract}
Acoustic and sonar analog signal processing applications require design of operational transconductance amplifiers (OTAs) that can be configured over wide frequency range in multiple bands and yet achieve low power consumption and low harmonic distortion. A fully differential, linear OTA is presented with digitally programmable transconductance ranging over three decades of dynamic range. Measurements from a prototype fabricated in a $0.5 \mu \mathrm{m}$ CMOS process demonstrate a $0.4 \mathrm{nA} / \mathrm{V}$ to $0.8 \mu \mathrm{A} / \mathrm{V}$ transconductance range, $40 \mathrm{~dB}$ common-mode rejection ratio (CMRR), and $-48 \mathrm{~dB}$ third-order harmonic distortion, at $12 \mu \mathrm{W}$ power dissipation.
\end{abstract}

\section{INTRODUCTION}

One of the advantages of field programmable analog arrays is its flexibility in reconfiguring circuit topologies and adapting them to specifications of different applications. In filter bank analog design, specifications may dictate adjustment of bandwidth parameters ranging over a wide range of frequencies covering audio to ultra-sonic range. The adjustment of filter parameters can be achieved either through digitally addressed capacitor arrays or through programmable OTAs whose transconductance can be digitally varied over a large range [1]. For very low frequency applications, digitally programmable capacitor sizing and resolution is limited by available silicon area, hence a more viable approach is to design wide range field programmable OTAs. Previous work in this field has focused on wide range analog tunability [2] using MOS transistors operating in subthreshold, or high-frequency applications [3] with tunability limited to 2 decades of transconductance. Limiting factors in increasing the range of tunability to several decades have been conflicting requirements of high linearity and dynamic range. This paper proposes an OTA architecture whose transconductance can be varied over 3 decades without appreciable degradation of its linearity or dynamic range. The design

This research was sponsored by ONR N00014-99-1-0612 and ONR/DARPA N00014-00-C-0315. The chip was fabricated through the MOSIS service.

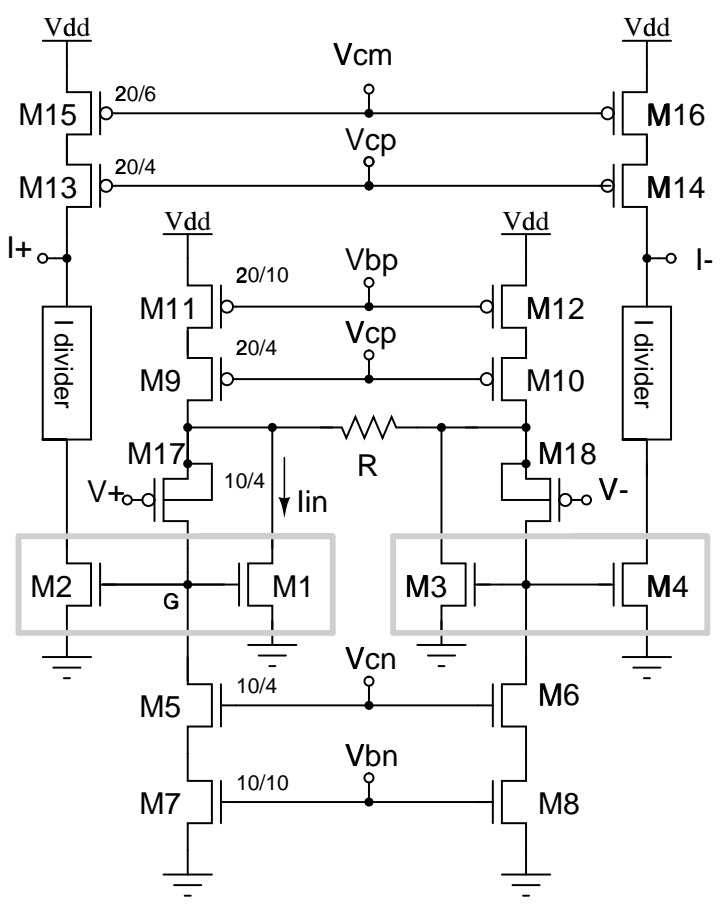

Fig. 1. Fully differential digitally programmable OTA. Transistor dimensions are given in units $\lambda=0.3 \mu \mathrm{m}$.

presented in this paper builds upon the tunable linear OTA architecture in $[4,1]$ and is augmented by using two-level digital current scaling techniques to obtain a wide-range linear transconductance.

The paper is organized as follows. Section 2 describes different elements used to design the digitally adjustable, fully differential OTA including current scaling techniques and common-mode feedback. Section 3 describes experimental results obtained from a prototype fabricated in $0.5 \mu$ CMOS technology, and section 4 provides concluding remarks. 


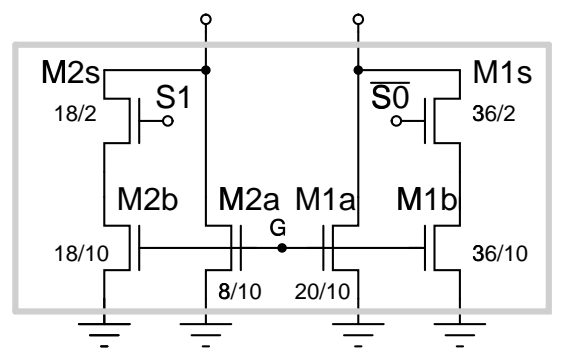

Fig. 2. Variable current ratioing for transistor pairs M1-M2 and M3-M4 in Figure 1.

\section{DIGITALLY PROGRAMMABLE OTA DESIGN}

Figure 1 shows the architecture of the OTA. The design is fully symmetric for high noise immunity and high power supply rejection ratio. The key elements in the design are the input transistors $M 17, M 18$, the current sensing transistors $M 1, M 3$ and the mirror transistors $M 2, M 4$. The current mirror ratios $M 1, M 2$ and $M 3, M 4$ are digitally selectable through bits $S_{1}$ and $S_{0}\left(\overline{S_{0}}\right)$ as clarified in Figure 2.

Denote $g m_{p}$ the transconductance and $g d_{p}$ the output conductance of the input stage transistors $M 17, M 18$. Also let $g m_{n}$ the transconductance of the current sensing transistors $M 1, M 3$. Then the relative current change $\delta I_{i n}$ with respect to a change in the differential input voltage $\delta V_{i n}$ is expressed as

$$
\delta I_{\text {in }} / \delta V_{\text {in }} \approx 1 / R
$$

where $R$ denotes the source degeneration linear resistance shown in Figure 1. Condition (1) is valid as long as

$$
R \gg 2 g d_{p} / g m_{p} g m_{n} .
$$

Rather than adjusting the linear resistance $R$ using active elements, it is more convenient to obtain a variable transconducance through scaling of the output current of the OTA first stage, in a second stage. Coarse level scaling is performed by the digitally programmable mirrors $M 1, M 2$ and $M 3, M 4$ and fine level scaling is performed at the output stage by the current divider in Figure 1, detailed in Figure 3.

\subsection{Current Divider}

The current divider scales the input current $I_{i n}$ by a binary coded digital factor with bits $B_{i}$, generating an output current:

$$
I_{\text {out }}=I_{\text {in }} \sum_{i=1}^{n} B_{i} 2^{-i}
$$

The current divider described in [6] and shown in Figure 3 offers the advantage of compactness in implementation, with four transistors for each bit of digital resolution.

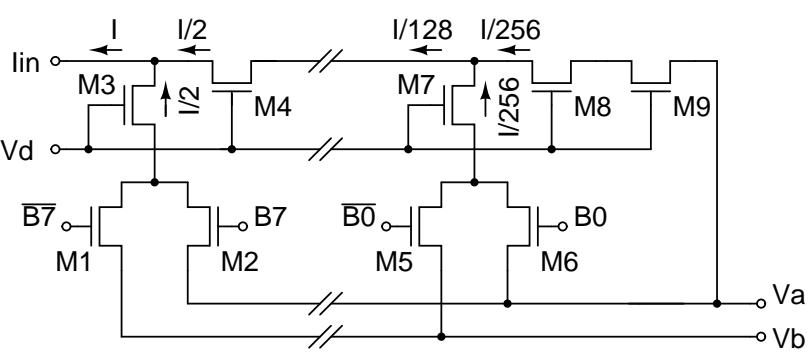

Fig. 3. 8-bit current divider [6]. All transistor are identically sized (8/8 $\lambda$ units).

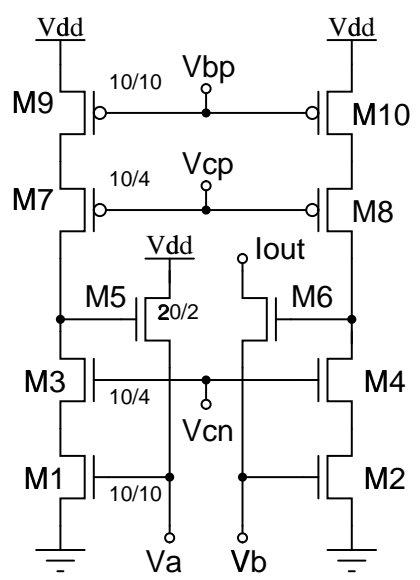

Fig. 4. Regulated cascode current conveyor for the current divider.

Critically important in achieving uniform scaling in the circuit of Figure 3 are low impedances nodes $V_{a}$ and $V_{b}$, ideally at same potential. This is achieved by using the regulated cascode current conveyor [7] architecture shown in Figure 4 . The node $I_{\text {out }}$ is connected to the output current source of the OTA as shown in Figure 1 to establish high output impedance as essential to a transconductance amplifier. The output common mode at this high impedances node is determined by a common-mode feedback circuit described below.

\subsection{Common Mode Feedback}

The common-mode stabilization for the OTA is achieved using the simple circuit shown in Figure 5. The circuit regulates the bias level $V_{c m}$ to maintain the maximum of $V^{+}$ and $V^{-}$to a predeterimined level, near the upper end of the range. Nonlinearity due to the maximum operation affect the outputs $V^{+}$and $V^{-}$, but not their difference to first order. In contrast to more involved circuits to maintain true common mode, e.g., [8], the common-mode circuit of Figure 5 uses fewer transistors and yet is effective. OTAs with common output nodes $V^{+}$and $V^{-}$share a single common- 


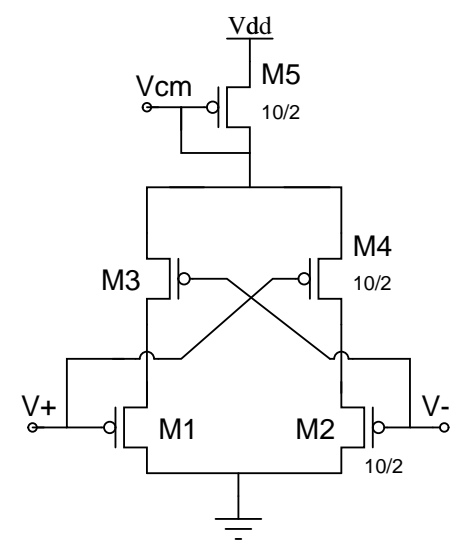

Fig. 5. Common-mode feedback circuit.

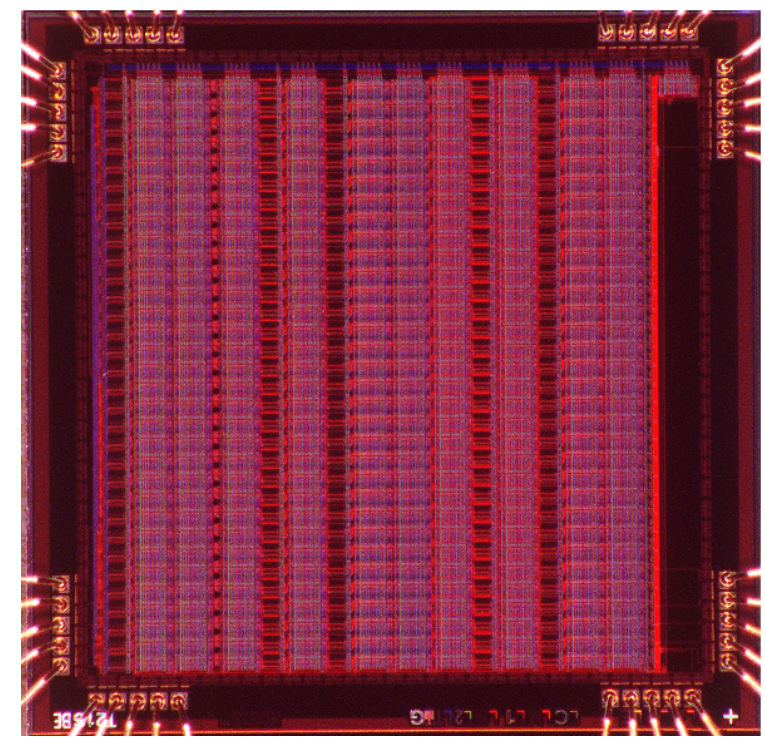

Fig. 6. Photomicrograph of programmable and reconfigurable OTA-C array.

mode stabilization circuit.

\section{EXPERIMENTS}

A prototype array of OTAs implementing a reconfigurable 32-channel filter-bank was fabricated in a $0.5 \mu$ CMOS process. The $3 \times 3 \mathrm{~mm}$ chip shown in Figure 6 consists of mainly 320 OTAs for different configurations of filter topologies.

Figure 7 presents linearity and dynamic range measurements from the OTA. The results indicate differential input dynamic range of $1.2 \mathrm{~V}$ and common-mode input range of $1.5 \mathrm{~V}$. The CMRR (common-mode rejection ratio) measures $40 \mathrm{~dB}$. Figure 8 demonstrates the wide digitally programmable range of the OTA. The four different curves

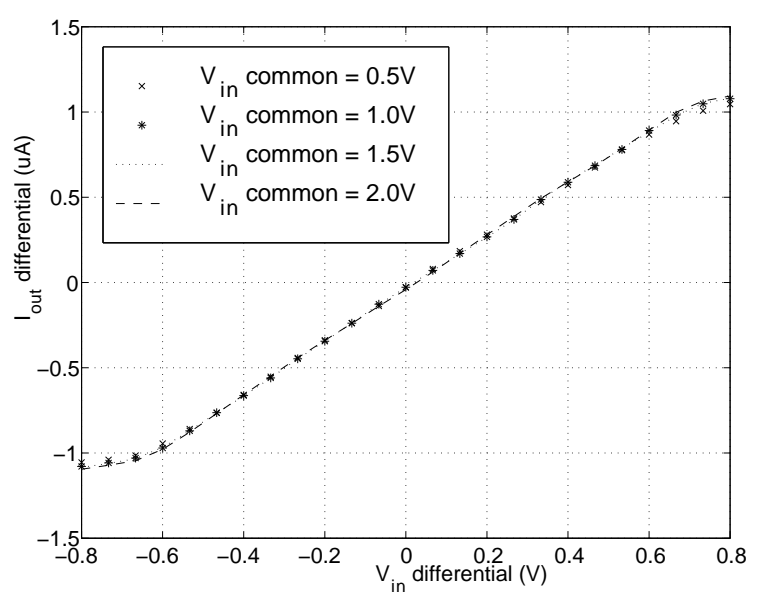

Fig. 7. Input differential and common-mode voltage range.

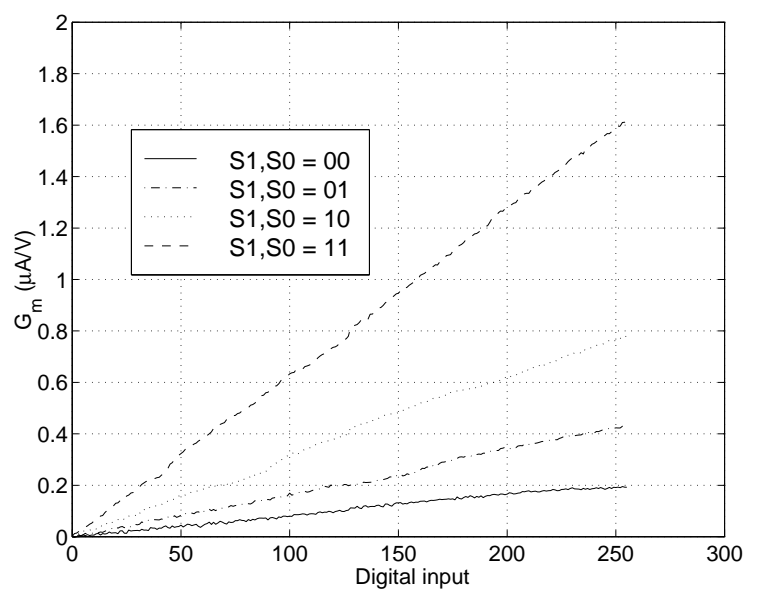

Fig. 8. Tuning curves: $G_{m}$ as a function of digital control bits B7...B0 and S1,S0.

shown correspond to different $S 1$ and $S 0$ bit settings which achieve coarse-level scaling by approximate factors 1, 2, 4 and 8 . Figure 8 also illustrates the effect of fine-level scaling indicated by the digital values in $x$ coordinates to achieve a scaling by a factor of 255 . In conjunction both stages of scaling result in an overall transconductance range of $0.39 \mathrm{nA} / \mathrm{V}$ to $0.8 \mu \mathrm{A} / \mathrm{V}$, covering more than three decades. Table 1 summarizes the overall specification and measured performance figures of a single OTA.

OTAs and capacitors can be combined on-chip to generate various filter topologies. As an example, a first-order low-pass filter was realized. The low-pass filter comprises two OTAs and a capacitor as shown in Figure 9. The filter was programmed to unity gain at low frequency (by programming the two OTAs to have identical transconductance value) and with variable cut-off frequency. The measured frequency response for various programmed values of the 


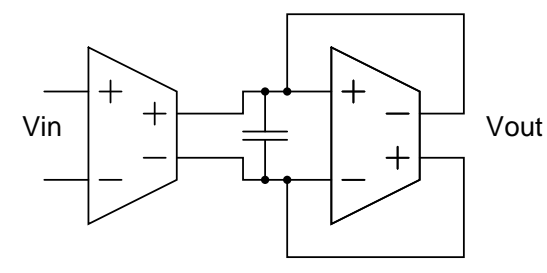

Fig. 9. Two OTAs and one capacitor configured as firstorder low-pass filter.

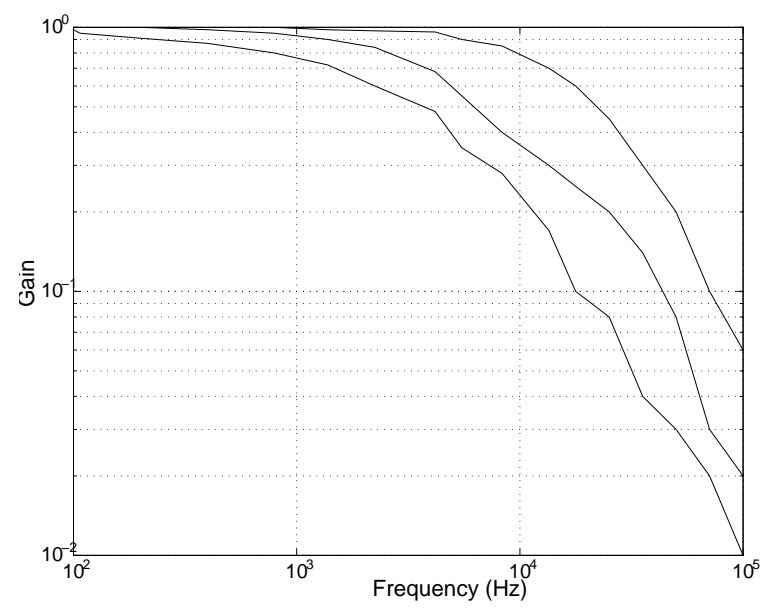

Fig. 10. Measured frequency response of first-order lowpass filter.

cut-off frequency is illustrated in Figure 10.

\section{CONCLUSION}

A fully differential operational transconductance amplifier with three decades of digitally programmable range has been presented. The OTA demonstrated low third order harmonic distortion, large CMRR and a large input dynamic range. The flexible design allows the OTA to operate with small bias currents which results in a minimum power consumption of $12 \mu \mathrm{W}$. This design can be adapted for use in different applications with center frequencies ranging from subsonic to ultrasonic frequencies $(10 \mathrm{~Hz}-120 \mathrm{kHz})$.

\section{REFERENCES}

[1] A. Chan Carusone and D. Johns, "A 5th Order Gm-C Filter in $0.25 \mu \mathrm{m}$ CMOS with Digitally Programmable Poles and Zeros," IEEE Int. Symp. Circuits and Systems (ISCAS'2002), Phoenix AZ, May 26-29, 2002.

[2] Paul M. Furth and Andreas G. Andreou, "Translinear Transconductor Design for Cochlear Filter Banks,"
Table 1. OTA Characteristics

\begin{tabular}{rl}
\hline Parameter & Measured Value \\
& \\
\hline Maximum $G m$ & $0.8 \mu \mathrm{A} / \mathrm{V}$ \\
Minimum $G m$ & $0.39 \mathrm{nA} / \mathrm{V}$ \\
Programming ratio & $1 / 2048$ \\
Input offset voltage & $20 \mathrm{mV}$ \\
Input dynamic range & $\pm 1.2 \mathrm{~V}$ \\
Third-order harmonic distortion & $-48 \mathrm{~dB}$ \\
Common-mode input voltage range & $0.5-3 \mathrm{~V}$ \\
Common-mode output voltage range & $1.0-4.0 \mathrm{~V}$ \\
Common-mode rejection ratio & $40 \mathrm{~dB}$ \\
Silicon area & $0.014 \mathrm{~mm}{ }^{2}$ \\
Power supply & $5 \mathrm{~V}$ \\
Power consumption & $12 \mu \mathrm{W}$ \\
& \\
\hline
\end{tabular}

Proc. 39th Midwest Symp. Circuits and Systems, pp. 917-920, Ames, IA, August 1996.

[3] B. Pankiewicz, M. Wojcikowski, S. Szczepanski and Yichuang Sun, "A field programmable analog array for CMOS continuous-time OTA-C filter applications" IEEE J. Solid-State Circuits, vol. 37 (2), Feb. 2002

[4] David A. Johns and Ken Martin, Analog Integrated Circuit Design, Wiley, New York, 1997.

[5] E. Sánchez-Sinencio and J. Silva-Martinez, "CMOS Transconductance Amplifiers, Architectures and Active Filters: a Tutorial" IEE Proc. Circuits Devices Syst., vol. 147 (1), Febr. 2000.

[6] Klaas Bult and Govert Gleelen, "An Inherently Linear and Compact MOST-Only Current Division Technique" IEEE Journal of Solid-State Circuits, vol. 27 (12), Dec. 1992.

[7] Sung Min Park and C. Toumazou, "A packaged lownoise high-speed regulated cascode transimpedance amplifier using 0.6m N-well CMOS technology" 26th European Solid-State Circuits Conference, Stockholm, Sweden, 19-21 Sept. 2000.

[8] Jaime E. Kardontchik, Introduction to the Design of Transconductor-Capacitor Filters, Kluwer Academic Publishers, 1992. 